\title{
Riesgo de Juego Patológico. Factores y trastornos mentales asociados en jóvenes de Medellín - Colombia
}

\section{Risk of Pathological Gambling, Associated factors and mental disorders in youth from Medellin - Colombia}

\author{
Mario A. Zapata; Yolanda Torres de G.; Liliana P. \\ MONTOYA
}

Grupo de Investigación en Salud Mental, Facultad de Medicina Universidad CES, Medellín, Colombia
Enviar correspondencia a:

Mario A. Zapata, Universidad CES-Facultad de Medicina,

Grupo de Investigación en Salud Mental, Calle 10A \#22-04 Medellín,

Colombia (SA). E-mail: mzapata@ces.edu.co

\section{RESUMEN}

Objetivos: Investigar el riesgo de juego patológico y su posible relación con trastornos mentales, problemas escolares y familiares, en una población escolarizada de la ciudad de Medellin (Colombia), para orientar la atención en salud mental.

Método: Estudio de corte transversal; muestra de 3.486 estudiantes de 10 a 19 años, probabilística y representativa de una población de 290.000 jóvenes. El instrumento SOGS (South Oaks Gambling Screening), fue utilizado para evaluar el riesgo y probabilidad de juego patológico; en etapa analítica se evaluó la posible asociación con trastornos mentales, uso de alcohol y drogas para juego patológico, descritas con significancia estadistica de $5 \%$ y proporciones de prevalencia con intervalos de confianza del 95\%

Resultados: $48,6 \%$ sin problemas, 37,6\% están en riesgo y $13,8 \%$ son posibles jugadores patológicos. Hombres tienen mayor riesgo que las mujeres $(2,5: 1)$ y la mayor proporción de posibles jugadores patológicos pertenecen al grupo de 10-14 años (58,7\%) vs. 15-19 (41,3\%). El Juego Patológico presenta asociación con ansiedad $R D=2,1(1,69-2,60)$, depresión $R D=1,73(1,33-2,24)$, intento de suicidio $R D=1,85(1,20-2,85)$, impulsividad $\mathrm{RD}=3,48(2,85-4,26)$, comportamiento violento $\mathrm{RD}=4,64(3,57-5,58)$, problemas en la escuela $\mathrm{RD}=2,46(1,97-3,06)$; el uso de cigarrillo, alcohol hasta la embriaguez y el uso-adicción a drogas ilegales, se identificaron como factores asociados con valores de $p<0,05$.

Conclusiones: La prevalencia de riesgo de juego patológico es alta para esta población y está asociada con trastornos mentales, consumo de sustancias psicoactivas y problemas en la relación familiar y escolar; esta situación señala la necesidad de generar un despliegue amplio e integral de intervención preventiva y terapéutica para impactar el problema.

Palabras clave: juego patológico, jóvenes, trastornos asociados, adicción recibido: Marzo 2010 aceptado: Junio 2010

\section{ABSTRACT}

Aims: To study the risk of pathological gambling and its possible relation with mental disorders and school and family problems in a population of school pupils from the city of Medellin (Colombia), with a view to providing guidance for mental health care.

Method:Cross-sectional study; sample comprises 3486 students aged 10 to 19 years selected from all the educational institutions in the city. The sample is probabilistic and represents an estimated population of 290,000 young people from Medellin. The SOGS instrument (South Oaks Gambling Screening) was used for screening risk and likelihood of problem gambling. The analytic stage assessed possible associated factors such as mental disorders, alcohol use and drug use, with statistical significance levels of $5 \%$ and prevalence rates with $95 \%$ confidence intervals.

Results: A total of $48.6 \%$ were without problems, $37.6 \%$ were at risk and $13.8 \%$ were possible pathological gamblers. Males were more at risk than females (2.5:1), and the highest proportion of possible pathological gamblers was in the 10-14 age group (58.7\%), vs. the 15-19 group (41.3\%). Pathological gambling in young people is associated in this study with: anxiety $\mathrm{OR}=2.1(1.69-2.60)$, depression $\mathrm{OR}=1.73(1.33-2.24)$, suicide attempt $O R=1.85(1.20-2.85)$, impulsivity $O R=3.48(2.85-4.26)$, violent behavior $\mathrm{OR}=4.64(3.57-5.58)$, and problems at school $\mathrm{OR}=2.46(1.97-3.06)$; recent use of cigarettes, drunkenness and illegal drugs (use/addiction) were identified as associated risk factors, $p$ value: $<0.05$

Conclusions: Pathological gambling risk is high in this young population, and is associated with mental disorders, use of psychoactive substances, and school and family problems. This situation suggests the need for largescale and comprehensive preventive and therapeutic intervention measures for addressing the problem.

Key Words: pathological gambling, youth, associated disorders, addiction. 


\section{INTRODUCCIÓN}

$\mathrm{E}$ riesgo de juego patológico en los jóvenes es hoy una preocupación para los gestores de las políticas en salud pública en el mundo, dado el aumento del problema y la mayor disponibilidad de información y discusión científica con la que se cuenta, sobre el impacto de la enfermedad en las diferentes esferas de desarrollo de los individuos (Jacobs, 1986; Messerlian, Derevensky y Gupta, 2005; Muñoz-Molina, 2008). Paralelamente a la preocupación por la enfermedad, que no es otra cosa que un efecto, surge la necesidad de estudiar e intervenir sus posibles causas, entre las cuales se ha señalado la mayor disponibilidad de juegos de apuestas en las últimas décadas, como factor facilitador de un mayor riesgo de juego patológico en la población. Estudios como el de Sullivan, McCormick y Sellman (1997) y Welte, Wieczorek, Barnes, y Tidwell (2006), sugieren la posible asociación entre el juego patológico y la nueva oferta de casinos.

Hoy se entiende y acepta al juego patológico como una enfermedad que debe ser tratada (Echeburúa, 2005; Ibáñez, Blanco, Donahue, Lesieur, Pérez de Castro, Fernández-Piqueras, et al. 2001) y la discusión ha evolucionado a cuál debe ser su ubicación categorial, si como trastorno del control de los impulsos o como adicción comportamental (APA, 2000; APA: DSM5, 2010); su interacción con otros problemas mentales y del comportamiento en los jóvenes, aunque de poca atención para la literatura psiquiátrica, merece mayor prioridad por el impacto que puede tener para el desarrollo de los adolescentes y de la estructura social y familiar en la que se desenvuelven (Bahamón, 2006; George y Murali, 2005; Kaminer y Petry, 1999; Korn y Shaffer, 1999).

Uno de los aspectos que se acepta como mediadores del riesgo de juego patológico en jóvenes, es la variada oferta disponible en las dos últimas décadas de juegos electrónicos y por internet; Petry (2006) en su estudio "internet gambling", encontró que esta práctica está relacionada con los comportamientos del juego patológico y señala en su estudio, que cerca de la mitad de aquellos quienes apostaron en internet, fueron clasificados como probables jugadores patológicos. De igual forma, demostró una asociación entre ser jugador patológico y problemas de salud física y mental para los individuos encuestados.

A la facilidad y rápido desarrollo de la tecnología de internet y los juegos electrónicos, se suma la proliferación de casinos en zonas urbanas, cuya disponibilidad e instalación aumenta aprovechando la facilidad y la desregulación que existe en algunos países (George y Murali, 2005). Estas nuevas formas de comercio son reconocidas como negocios legítimos, que la sociedad en general aprueba, pero con poco análisis de sus consecuencias (Andresen, 2006).

En la última década, se han publicado estudios que muestran la rápida expansión del problema y la afectación que puede tener la población a través del incremento del número de jugadores patológicos y problemas relacionados (Griffiths, 2004). En Norteamérica, estudios estiman que 15.3 millones de adolescentes realizan actividades de juegos de apuestas y de estos 2.2 millones tiene problemas con el juego o son considerados jugadores patológicos (Jacobs, 2000); en Canadá, en la encuesta nacional del año 2002, el 61.35\% de jóvenes entre 15 y 24 años tuvo contacto con juego de apuestas en el último año y el $2.2 \%$ del total de la población en este grupo se considera en riesgo moderado o jugador patológico (Huang y Boyer, 2007). En España, en descripciones de Arbinaga (2000), se señalan prevalencias de Juego Patológico en menores que oscilan entre 1,6\% y 2,9\%. En Latinoamérica no se encuentran estudios de prevalencia poblacional del problema (Muñoz-Molina, 2008) pero en Colombia estudios han explorado las conductas en niños y jóvenes que pueden ser de riesgo para juego patológico (Ballesteros, Novoa, Caycedo y García, 2006).

La situación epidemiológica del Juego Patológico, obliga a priorizar su atención a través de acciones de prevención y tratamiento, pero con una visión de integralidad en su abordaje. Este abordaje integral, coloca la evaluación del juego patológico dentro de un contexto ecológico multicausal (Bronfenbrenner, 1986; Jacobs, 1986; McLeroy, Bibeau, Steckler y Glanz, 1988). El contexto ecológico tiene que ver con las características individuales, la familia, la sociedad, sus normas y relaciones, que como resultado final puede afectar el desempeño individual a nivel educativo, laboral y de comportamiento en el entorno inmediato del individuo; en este contexto juega un papel importante la comorbilidad que el jugador patológico puede tener con otros trastornos mentales como, ansiedad, trastornos afectivos, impulsividad, abuso de drogas y alcohol, cuya posible asociación ha sido documentada en otros estudios (Ciarrocchi y Richardson, 1989; Jacobs, 1986; Lynch, Maciejewski y Potenza, 2004; Vitaro, Arseneault y Tremblay, 1997). La evidencia genética que favorece el riesgo de juego patológico en hijos de padres jugadores y el posible compromiso en actividades delictivas del jugador patológico como medio para sostener su actividad en el juego, enmarca este trastorno en un espectro amplio y variado de relaciones y causas de no fácil intervención (Black, Monahan, Temkit y Shaw, 2006; García, Díaz y Aranda, 1993; Lobo y Kennedy, 2009).

La consideración del crecimiento y reconocimiento de la enfermedad en el mundo y de sus efectos en la salud de la población y de los jóvenes, obliga a decisores de política pública, investigadores, terapeutas e instituciones de tratamiento, a promover el acceso al mejor conocimiento del problema, a estudiar la prevalencia de la enfermedad y los factores de riesgo en cada uno de los países y a definir las mejores prácticas en tratamiento. Lo anterior enmarcado en la necesidad de formular una política pública para el juego patológico (Shaffer, 2003), la cual deberá nacer de una caracterización del problema que permita entenderlo y acercarse a su relación causal y que lleve a decisiones correctas para su manejo. Bajo esta perspectiva, este estudio tiene como objetivo obtener un buen conocimiento inicial del riesgo de juego patológico, aplicado a un porcentaje muy alto de la población adolescente de una ciudad capital Latinoamericana, para este caso Medellín (Colombia); permitirá además probar instrumentos de medición que han tenido éxito en otras regiones y en otros idiomas, entregando como resultado secundario, el análisis de validez y fiabilidad que permita 
que estas mediciones puedan realizarse con éxito en otros países Suramericanos y se cuente con evidencia regional estandarizada y comparable para definir acciones que puedan impactar positivamente el problema.

\section{MÉTODO}

Estudio de corte transversal; en una primera etapa desarrollo descriptivo y una posterior analítica que utiliza el análisis bivariado y la medición de Razones de Disparidad $(R D=0 R)$, para la identificación de posibles asociaciones entre el riesgo de juego patológico con factores como, el funcionamiento familiar, la adaptación escolar, trastorno de ansiedad, depresión, suicidio, comportamiento violento, impulsividad, uso de drogas, alcohol y cigarrillo.

\section{Población y Muestra}

La muestra corresponde a 3.486 estudiantes de secundaria entre 10 y 19 años de edad, seleccionados de forma aleatoria y representativa del total de los estudiantes matriculados en los centros educativos de secundaria de la ciudad de Medellín-Colombia. La muestra es probabilística y representa una población estimada de 290.000 jóvenes, población que para la ciudad, corresponde al $87,5 \%$ del total en este grupo etáreo (DANE, 2009).

El tamaño se calculó con base en la proporción de una población, utilizando un nivel de significancia del 5\%, una precisión del 4\%, una prevalencia estimada para trastornos por consumo de drogas de $15 \%$ y una corrección por efecto de diseño de 4.

\section{Instrumentos}

El instrumento SOGS (South Oaks Gambling Screening) desarrollado por Lesieur y Blume (1987), fue utilizado con la autorización de sus autores previa adaptación al lenguaje de la región. Fue seleccionado por su utilización previa en investigaciones similares para medir el riesgo de juego patológico, lo cual es valioso para la comparabilidad de los resultados (Lesieur y Blume, 1993). SOGS es un instrumento de "screening" compuesto por 20 ítems basado en los criterios diagnósticos de juego patológico del DSM III y del DSM III-R (American Psychiatric Association); ha sido catalogado como el instrumento más fiable y válido de que se dispone en la actualidad para evaluar el juego patológico en los estudios epidemiológicos y clínicos (Echeburúa, Báez, Fernández y Páez, 1994; Stinchfield, 2002).

Con base en este instrumento, se construyó la primera versión de la encuesta de recolección con 19 variables específicas para identificar el riesgo de tener problemas con el juego; a través de la prueba alfa de Cronbach con resultado de 0,84 , se demostró una buena fiabilidad y consistencia interna del instrumento (George y Mallery, 1995). Este valor es cercano al resultado de 0,94 de la validación española (Echeburúa, et al. 1994) y del resultado de 0,97 de la validación original de Lesieur y Blume (1987).

Para el análisis se utilizó la clasificación original propuesta por los autores del instrumento SOGS, esto es, puntuación 0 "sin problemas", 1 a 4 "riesgo o algunos problemas" y 5 o más "probable jugador patológico", más una clasificación dual propuesta para este estudio y enriquecer el análisis de los resultados bivariados, en donde se denomina "sin problema" a la puntuación 0 y se denomina "riesgo general" a la puntuación mayor a 1, la cual comprende dos niveles de riesgo, algunos problemas y jugador patológico. Esta agrupación ha sido utilizada en otras investigaciones para el estudio del riesgo de juego patológico en adolescentes (Shaffer y Hall, 1996; Winters, Stinchfield, Botzet y Anderson, 2002). La primera versión de la encuesta se puso a prueba en una muestra piloto y se elaboró el segundo y definitivo instrumento, el cual tiene semejanza a la validación española, versión que fue muy útil en desarrollo del estudio (Echeburúa, et al. 1994).

Otras escalas en salud mental, uso y adicción a sustancias psicoactivas fueron utilizadas en la investigación para la exploración y búsqueda de posible asociación con los problemas de adicción al juego. Estas escalas han sido utilizadas y demostrada su fiabilidad y validez, en otros estudios que ha desarrollado el Grupo de Salud Mental de la Facultad de Medicina de la Universidad CES en población similar (Maya, Torres, Murrelle, Rodríguez, Mejía, Gaviria, et al. 2000; Torres, Osorio, López y Mejía, 2006); entre ellas se destacan: CDI, escala de depresión en niños de Kovacs (1985); CMAS$R$, escala de ansiedad en niños revisada de Reynolds y Richmond (1978); DUSI, inventario diagnóstico del uso de drogas y la posible adicción a drogas legales e ilegales (Tarter, 1990). En este último, se exploró el consumo más próximo de alcohol y cigarrillo, esto es, último día y última semana, bajo la hipótesis que quienes reportan un consumo reciente posiblemente son aquellos que más se acercan a la definición de ser consumidores habituales de la sustancia psicoactiva; se utilizó además la subescala DUSI que mide la dimensión problemas académicos (Tarter, 1990; Tarter, Kirisci y Mezzich, 1996). La exploración del riesgo de suicidio se valoró con el apoyo de las definiciones del instrumento CIDI, OMS (1990) y para la medición del maltrato se utilizó instrumento validado por Maya et al. (2000) y el cual considera las formas de maltrato verbal, físico o sexual. Sus variables y opciones de respuesta están diseñadas de manera tal, que el encuestado deba responder frente a situaciones indirectas de maltrato en el hogar lo cual en hipótesis pudo ocurrir con él mismo; la forma indirecta de la pregunta pretende mejorar la veracidad de la información que se entregue sobre el tema.

\section{Procedimiento}

La recolección se realiza mediante la aplicación de instrumento autoadministrado de 55 variables, con el soporte 


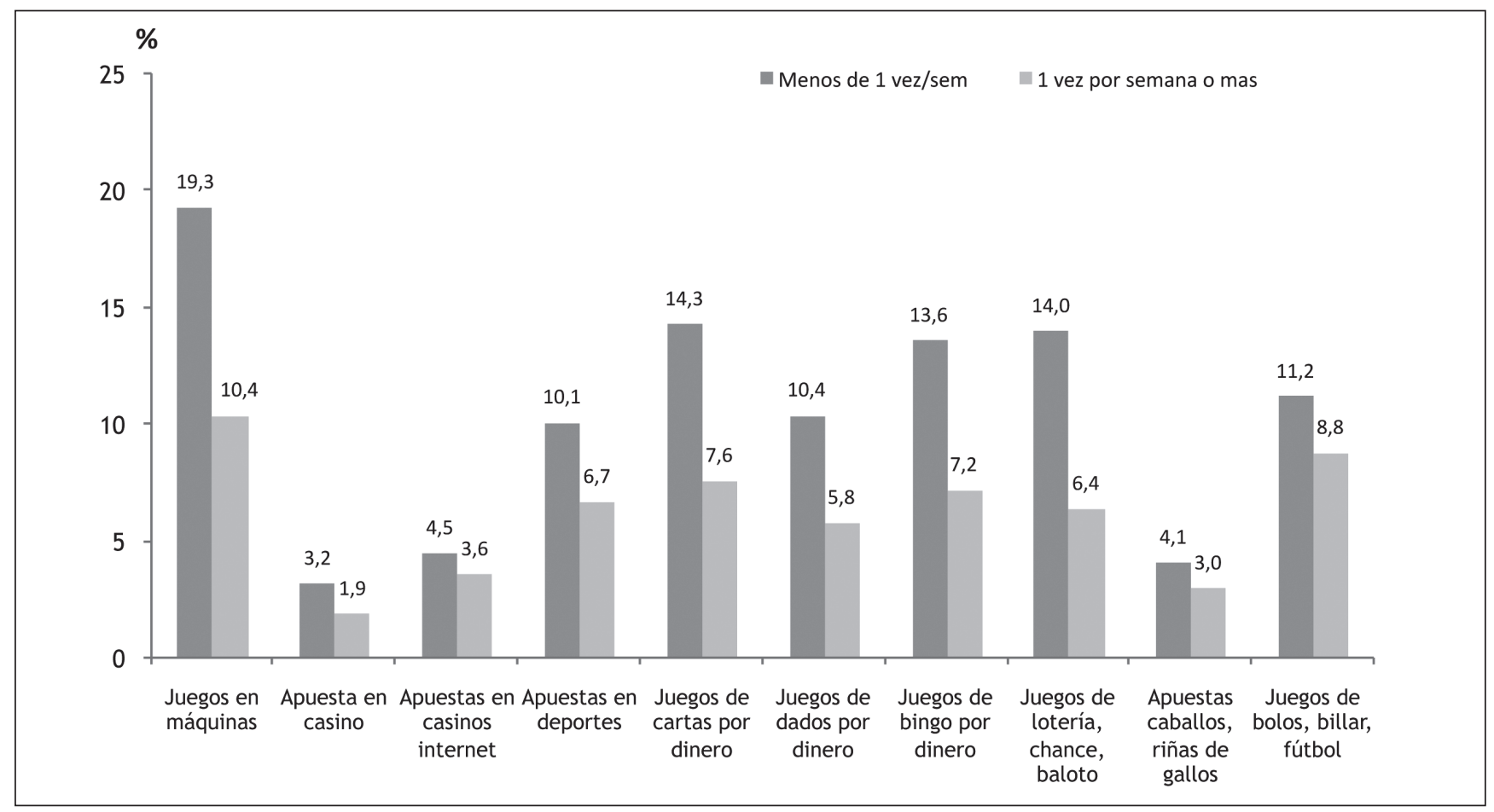

Figura 1. Distribución según tipos de apuestas en las que los jóvenes han participado.

de un equipo de psicólogos previamente entrenados. El instrumento de recolección se preparó en el programa Teleform ${ }^{\circledR}$, lo que permitió la captura electrónica de los datos, previa la revisión manual crítica de la información, generando así la base de datos que luego se exportó al programa SPSS (Copyright ${ }^{\circledR}$ SPSS Inc) para el cálculo de los indicadores.

De acuerdo a los objetivos del estudio, en una primera etapa se obtienen medidas descriptivas de la población estudiada, de las variables sociodemográficas, de los indicadores de salud mental y de riesgo de juego patológico, identificando su prevalencia con intervalos de confianza de 95\%. Luego se estimaron medidas de asociación RD (razón de disparidad) con IC 95\% entre los factores estudiados y el juego patológico. Igualmente se llevó a cabo la estimación del comportamiento de la posible comorbilidad entre juego patológico y otros trastornos mentales: ansiedad, depresión, riesgo de suicidio y consumo de sustancias psicoactivas.

\section{RESULTADOS}

La distribución proporcional hombre-mujer en este estudio en la ciudad de Medellin, permite explorar y concluir sobre la situación del riesgo de juego patológico para ambos sexos y su comparación entre ellos. El 56,9\% (1984) mujeres y $43,1 \%$ (1502) hombres fueron encuestados.

La primera pregunta utilizada en el instrumento de evaluación SOGS, permitió la identificación de los juegos de apuestas disponibles, a las que han estado o están expues- tos los jóvenes en esta región. Su descripción es importante porque la facilidad en el acceso a estas opciones de juego, puede ser la puerta de entrada para generar conductas de riesgo o de juego patológico.

En la figura 1, se muestra que los juegos en donde los jóvenes apuestan con mayor frecuencia son: juegos de máquinas (10,4\%), juegos de bolos, billar, futbol $(8,8 \%)$ y juego de cartas (7,6\%). Las apuestas en casino a pesar de su restricción legal a menores de edad, son reportadas 1,9\% en la categoría de una vez por semana o más y las apuestas en casinos por internet son del 3,6\% para esta misma categoria de frecuencia. Otro tipo de apuestas observadas aparentemente inocuas por los adultos como las apuestas en juego de bingo, ocupan un lugar importante en las preferencias de juego de los jóvenes.

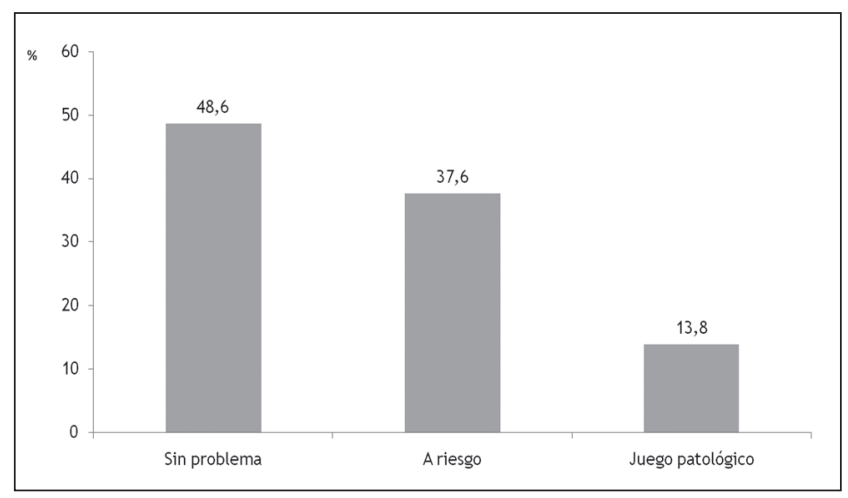

Figura 2. Distribución de la prevalencia de riesgo y probabilidad de Juego Patológico. 
Por primera vez en Colombia y en Sur América se aplica el instrumento SOGS en una muestra amplia y representativa de jóvenes de una ciudad capital. Según lo muestra la figura 2, el 48,6\% de jóvenes encuestados no tiene problemas con el juego, 37,6\% presenta riesgo o algunos problemas y el $13,8 \%$ del total de la población son probables jugadores patológicos.

Con respecto a la distribución del riesgo o la probabilidad de juego patológico según sexo, la tabla 1 muestra en los hombres una mayor proporción para la categoría de probables jugadores patológicos en comparación con las mujeres (71,4\% hombres versus $28,6 \%$ en mujeres) presentándose una relación 2,5:1. Para el caso de los jóvenes en riesgo, es ligeramente mayor la proporción de mujeres en riesgo $(52,1 \%$ versus $47,9 \%)$. Estas diferencias de proporciones fueron estadísticamente significativas.

Tabla 1. Distribución de la escala de riesgo de Juego Patológico según sexo.

\begin{tabular}{|l|c|c|c|c|c|c|c|c|}
\hline & \multicolumn{5}{|c|}{ ESCALA DE JUEGO PATOLÓGICO } & & \multirow{2}{*}{ Total } & $\%$ \\
\hline & $\begin{array}{c}\text { Sin } \\
\text { problema }\end{array}$ & $\%$ & A Riesgo & $\%$ & $\begin{array}{c}\text { Probable } \\
\text { Jugador } \\
\text { Patológico* }\end{array}$ & $\%$ & & \\
\hline Hombre & 508 & 30,0 & 627 & 47,9 & 344 & 71,4 & 1479 & 42,42 \\
\hline Mujer & 1186 & 70,0 & 683 & 52,1 & 138 & 28,6 & 2007 & 57,57 \\
\hline Total & 1694 & 100 & 1310 & 100 & 482 & 100 & 3486 & 100 \\
\hline
\end{tabular}

* valor de $\mathrm{p}:<0.05$ diferencias significativas

\section{Análisis Bivariado}

Se pretende indagar sobre las posibles asociaciones causales que el riesgo y la probabilidad de ser jugador patológico, pueden tener con otros trastornos mentales y con el consumo o la adicción a sustancias psicoactivas; además la posible asociación con situaciones como: relación con el maestro, problemas académicos, cohesión familiar, maltrato, ideación-plan o intento de suicidio.

\section{Diferencias encontradas en e/ sexo y grupos de edad}

En la tabla 2 se observa que los hombres tienen un riesgo mayor a las mujeres, para las variables respuesta riesgo general de ser jugador patológico y jugador patológico. Comparado el grupo de edad 10 a 14 años con el de 15 a 19, se muestra como posible factor de riesgo para ser Jugador patológico con una RD:1,04, pero las diferencias no fueron estadísticamente significativas; situación diferente se presenta cuando el análisis se realiza con respecto al riesgo general definido en este estudio, donde la RD:0,84 muestra como factor protector pertenecer al grupo de 10 a 14 años, con diferencias estadísticas significativas. Este resultado puede interpretarse como la existencia de mayor riesgo para presentar problemas con el juego en el grupo de 15 a 19, lo cual parece lógico por el mayor acceso que este grupo de edad puede tener a los sitios de juegos, bien sea virtuales 0 presenciales.

Tabla 2. Análisis bivariado. Exposición a factor sexo y grupo de edad y riesgo de Juego Patológico en jóvenes de 10 a 19 años.

\begin{tabular}{|l|c|c|c|c|}
\hline Variable exposición vs. Resultado & RD & IC & $\chi^{2}$ & Valor de $p$ \\
\hline 1. Sexo Masculino: & & & & \\
- Jugador patológico & 4,10 & $(3,32-5,07)$ & 191,81 & 0,0001 \\
- Riesgo general JP* & 2,76 & $(2,40-3,17)$ & 208,73 & 0,0001 \\
\hline 2. Grupo de edad 10 a 14 años*: & & & & \\
- Jugador patológico & 1,04 & $(0,86-1,27)$ & 0,169 & 0,6806 \\
- Riesgo general JP* & 0,84 & $(0,74-0,97)$ & 5,97 & 0,0146 \\
\hline
\end{tabular}

* Riesgo general JP: suma de categorías de "' algún problema" y "Jugador Patológico".

** Grupo de comparación 15 a 19.

\section{Asociación con trastornos mentales, suicidio}

Los resultados de la tabla 3 muestran que la ansiedad $(R D: 2,1)$, la impulsividad $(R D: 3,48)$, el comportamiento violento $(R D: 4,64)$ y la irritabilidad (RD:2,65), están asociadas con significancia estadística para el resultado de probable jugador patológico en los jóvenes. Igual resultado de asociación aunque con valores de RD menores, se presenta para la definición de riesgo general de juego patológico. La depresión se encuentra asociada con el riesgo de ser jugador patológico con resultados estadísticamente significativos (RD:1,73, valor de $p<0,05)$; cuando se evalúa con el riesgo general modelado en este estudio, se encuentran valores que no muestran significancia estadística RD:0,94 (IC:0,77-1,15) y valor de $p>0,05$. La aparente contradicción en el hallazgo, puede leerse como la asociación de la depresión con el diagnóstico de juego patológico pero no necesariamente de su asociación con niveles inferiores de riesgo.

Tabla 3. Análisis bivariado. Exposición a factor trastornos mentales y riesgo de Juego Patológico en jóvenes de 10 a 19 años.

\begin{tabular}{|l|c|c|c|c|}
\hline Variable exposición vs. Resultado & RD & IC & $\chi^{2}$ & Valor de $p$ \\
\hline 1. Ansiedad: & & & & \\
- Jugador patológico & 2,10 & $(1,69-2,60)$ & 46,84 & 0,0001 \\
- Riesgo general JP* & 1,25 & $(1,09-1,43)$ & 10,15 & 0,0014 \\
\hline 2. Depresión: & & & & \\
- Jugador patológico & 1,73 & $(1,33-2,24)$ & 17,54 & 0,0001 \\
- Riesgo general JP* & 0,94 & $(0,77-1,15)$ & 0,400 & 0,5270 \\
\hline 3. Impulsividad: & & & & \\
- Jugador patológico & 3,48 & $(2,85-4,26)$ & 159,60 & 0,0001 \\
- Riesgo general JP* & 2,27 & $(1,97-2,61)$ & 129,46 & 0,0001 \\
\hline 4. Irritabilidad: & & & & \\
- Jugador patológico & 2,65 & $(2,15-3,25)$ & 89,98 & 0,0001 \\
- Riesgo general JP* & 1,73 & $(1,52-1,98)$ & 64,63 & 0,0001 \\
\hline 5. Comportamiento violento: & & & & \\
- Jugador patológico & 4,64 & $(3,57-5,58)$ & 195,70 & 0,0001 \\
- Riesgo general JP* & 2,69 & $(2,34-3,08)$ & 200,51 & 0,0001 \\
\hline
\end{tabular}

* Riesgo general JP: suma de categorías de "'algún problema" y "Jugador Patológico". 
Para el análisis de la posible asociación con el suicidio, se exploraron las dimensiones de riesgo de: ideación, plan suicida, intento e intento con necesidad de atención en salud. Como resultado en la tabla 4 se muestra que aquellos jóvenes en quienes se detecta alguno(s) de los cuatros niveles de riesgo de suicidio, presentan asociación con el riesgo de ser jugador patológico. La mayor magnitud de asociación se reporta en quienes intentaron el suicidio y necesitaron atención en salud.

Tabla 4. Análisis bivariado. Exposición al factor dimensiones del Suicidio y riesgo de ser Jugador Patológico, en jóvenes 11 a 19 años.

\begin{tabular}{|l|c|c|c|c|}
\hline Variable exposición vs. Resultado & RD & IC & $\chi^{2}$ & Valor de $\mathbf{p}$ \\
\hline 1. Suicidio y Jugador Patológico*: & & & & \\
- Ideación & 1,48 & $(1,20-1,83)$ & 13,79 & 0,0002 \\
- Plan & 1,55 & $(1,20-1,99)$ & 11,62 & 0,0001 \\
- Intento & 1,47 & $(1,15-1,88)$ & 9,37 & 0,0022 \\
- Intento (necesitó atención salud) & 1,85 & $(1,20-2,85)$ & 8,06 & 0,0045 \\
\hline
\end{tabular}

${ }^{*}$ Riesgo de ser Jugador Patológico

\section{Cigarrillo, alcohol y drogas ilegales}

La tabla 5 muestra resultados estadísticamente significativos de asociación entre consumo de cigarrillo o alcohol y la probabilidad de ser jugador patológico o tener riesgo general. Quienes han consumido alcohol hasta la embriaguez en el último día y/o la última semana, presentan riesgo de ser probables jugadores patológicos con una RD:3,10 y un riesgo general con una $\mathrm{RD}: 2,68$. Se resalta que estos resultados de asociación, son mayores que los del consumo sin embriaguez; igualmente, aplicada la subescala DUSI de posible dependencia al alcohol, el resultado muestra que existe asociación estadística significativa entre el riesgo de tener dependencia al alcohol y ser jugador patológico (RD:2,69) y similar resultado se encuentra con el riesgo general (RD:2,08).

La posible dependencia a las drogas ilegales se encontró con asociación estadísticamente significativa para los riegos definidos de juego patológico.

Tabla 5. Análisis bivariado. Exposición al factor uso de alcohol, drogas y posible dependencia y riesgo de Juego Patológico, en jóvenes de 10 a 19 años.

\begin{tabular}{|l|c|c|c|c|}
\hline Variable exposición vs. Resultado & RD & IC & X2 & Valor de $p$ \\
\hline 1. Cigarrillo: & & & & \\
- Jugador patológico & 2,46 & $(1,95-3,12)$ & 52,15 & 0,0001 \\
- Riesgo general JP* & 2,29 & $(1,86-2,81)$ & 63,52 & 0,0001 \\
\hline 2. Alcohol: & & & & \\
- Jugador patológico & 2,11 & $(1,71-2,59)$ & 51,75 & 0,0001 \\
- Riesgo general JP* & 1,93 & $(1,64-2,28)$ & 63,79 & 0,0001 \\
\hline 3. Alcohol Embriaguez: & & & & \\
- Jugador patológico & 3,10 & $(2,33-4,11)$ & 66,07 & 0,0001 \\
- Riesgo general JP* & 2,68 & $(2,02-3,55)$ & 50,31 & 0,0001 \\
\hline 4. DUSI ALCOHOL: & & & & \\
- Jugador patológico & 2,69 & $(2,21-3,28)$ & 102,28 & 0,0001 \\
- Riesgo general JP* & 2,08 & $(1,81-2,39)$ & 108,22 & 0,0001 \\
\hline 5. DUSI DROGAS: & & & & \\
- Jugador patológico & 2,96 & $(2,36-3,70)$ & 195,70 & 0,0001 \\
- Riesgo general JP* & 2,26 & $(1,85-2,76)$ & 67,01 & 0,0001 \\
\hline
\end{tabular}

Medición: Instrumento DUSI. * Riesgo general JP: suma de categorias de "'algún problema" y "Jugador Patológico".

\section{Otros Factores: maltrato, la escuela, la familia}

Evaluados el maltrato en el hogar, las malas relaciones con el maestro y los malos resultados académicos, se encontraron asociaciones estadísticamente significativas con juego patológico y con el riesgo general. Finalmente el estudio evaluó las relaciones con la familia como posible factor favorecedor o protector para el riesgo de juego patológico y se obtuvo que la cohesión familiar mala o regular, es factor de riesgo para que los jóvenes sean jugadores patológicos; para el resultado riesgo general, en donde se tienen en cuenta la suma de los riesgos, "posibles problemas" y "jugador patológico", el resultado muestra que no hay asociación RD:1,09 $(0,83-1,42)$. Lo anterior puede justificarse en el mayor peso que otros factores puede tener para el riesgo de ser jugador patológico, pero este análisis supera el alcance de la metodología propuesta en este estudio.

Tabla 6. Análisis bivariado. Exposición al factor Maltrato, Escuela, Familia y riesgo de Juego Patológico, en jóvenes de 10 a 19 años.

\begin{tabular}{|l|c|c|c|c|}
\hline Variable exposición vs. Resultado & RD & IC & $\chi^{2}$ & Valor de $p$ \\
\hline $\begin{array}{l}\text { 1. Maltrato: } \\
\text { - Jugador patológico } \\
\text { - Riesgo general JP* }\end{array}$ & 2,47 & $(1,97-3,11)$ & 62,87 & 0,0001 \\
\hline 2. Problemas Académicos: & 1,46 & $(1,20-1,76)$ & 15,19 & 0,0001 \\
- Jugador patológico & 2,46 & $(1,97-3,06)$ & 67,58 & 0,0001 \\
- Riesgo general JP* & 1,53 & $(1,34-1,75)$ & 38,76 & 0,0001 \\
\hline 3. Relación con el Maestro: & & & & \\
- Jugador patológico & 1,78 & $(1,46-2,17)$ & 32,69 & 0,0001 \\
- Riesgo general JP* & 1,21 & $(1,06-1,38)$ & 8,11 & 0,0044 \\
\hline 4. Cohesión Familiar: & & & & \\
- Jugador patológico & 2,01 & $(1,45-2,78)$ & 18,56 & 0,0001 \\
- Riesgo general JP* & 1,09 & $(0,83-1,42)$ & 0,38 & 0,5368 \\
\hline
\end{tabular}

* Riesgo general JP: suma de categorías de "'algún problema" y "Jugador Patológico".

\section{DISCUSIÓN}

El análisis de los resultados de riesgo de juego patológico en los jóvenes, emerge con gran interés para la toma de decisiones urgentes en salud pública para impactar el problema. Pocas mediciones existen en Colombia y en los países Suramericanos y esta carencia lleva consigo falta de política y prioridad pública.

Algunos resultados por su magnitud, sorprenden a los investigadores. El 13,8\% de los jóvenes presentan el más alto riesgo descrito en el instrumento de medición SOGS, "probable jugador patológico"; este valor supera ampliamente los hallazgos encontrados en otras investigaciones y regiones del mundo, lo cual puede explicarse por el gran aumento en los últimos años de sitios legales e ilegales de juegos de apuestas en la ciudad de Medellín según diagnóstico presentado por Gutiérrez (2010). Jacobs (1989), encontró prevalencias en adolescentes entre el 4 y 6\%, Shaffer y Hall (1996) en su revisión estiman prevalencia entre 4 y $7 \%$ y Arbinaga (2000) encontró en su estudio descriptivo con estudiantes de 8 a 
17 años una prevalencia de 5,8\%. Bajo esta consideración, la cifra de posibles jugadores patológicos en jóvenes de la ciudad de Medellín duplica las mayores cifras encontradas en otros estudios. Vale la pena señalar que la medición en esta investigación se hizo con el punto de corte más exigente de la escala, esto es, 5 o más para probables jugadores patológicos, valor propuesto originalmente por los autores del instrumento (Lesieur y Blume, 1987) y que supera el punto de corte de 4 propuesto por la validación española (Echeburúa, et al. 1994).

Existe acuerdo en los diferentes estudios, que los hombres están más expuestos al riesgo de juego patológico. La relación encontrada en este estudio de 2,5:1 (hombremujer), se acerca a lo reportado por investigadores en diferentes culturas (Adlaf y lalomiteanu, 2000; Ko, Yen, Chen, Chen y Yen, 2005; Westphal, Rush, Stevens y Johnson, 2000). Pietrzack, Ladd y Petry (2003), señalan al sexo masculino como factor de riesgo para el juego patológico y otros estudios, entre ellos el de Huang y Boyer (2007), han encontrado una razón de 3:1.

Una discusión reciente y planteada en la revisión del DSM-IV-TR, para la actualización y expedición del DSM-V, es la categoría que debe ocupar el juego patológico como trastorno mental. Desde su inclusión en 1980 en el DSM-III, el Juego patológico fue ubicado en los desordenes del control de los impulsos, pero los recientes avances en su estudio, lo han llevado a ser propuesto en la categoría de adicción no química, esto es, no a sustancias, sino como resultado de una modificación mórbida del comportamiento. En aporte a esta discusión se encontró que la impulsividad, junto con el comportamiento violento, son los trastornos mentales que muestran mayor magnitud de asociación con el Juego Patológico y la ansiedad igualmente, muestra una asociación como factor de riesgo para este problema. En consideración a este hallazgo, una nueva clasificación del juego patológico como adicción, no debe subvalorar su posible origen en el trastorno control de los impulsos y en el manejo terapéutico que este requiere.

Comentario especial merecen los hallazgos de comorbilidad encontrados en lo que se refiere a la depresión. De manera consistente, los diferentes trabajos la señalan como uno de los trastornos mentales asociados con el riesgo de juego patológico (Becona, Lorenzo y Fuentes, 1996; McCormick, Russo, Ramirez y Taber, 1984; Westphal y Johnson, 2003). El presente estudio tiene resultados similares en esta dirección en lo que se refiere al riesgo de ser Jugador Patológico, mas no para la exploración general que se hizo en donde se agruparon los dos niveles de riesgo (algún problema y jugadores patológicos). Este hallazgo puede explicarse cómo, la mayor probabilidad que la depresión se exprese con preferencia en aquellos que tienen niveles más graves de psicopatología con el juego.

De otra parte, es consistente el hallazgo de asociación de las dimensiones de riesgo del suicidio con el riesgo de ser jugador patológico; este resultado es similar al de otros estudios, en donde la ideación y los intentos suicidas han sido identificados como asociados a los jugadores patológicos (Arbinaga, 2000; George y Murali, 2005; Ibáñez, Blanco,
Donahue, Lesieur, Pérez de Castro, Fernández-Piqueras, et al. 2001). Similar situación se encuentra con el consumo de alcohol como factor asociado a esta patología; ser consumidor de alcohol y de alcohol hasta la embriaguez se reporta en este estudio como riesgo, con uno de los valores de asociación más altos (RD: 3,1$)$. Esta situación también es descrita por Baez y Echeburúa (1994), los cuales afirman que el consumo abusivo de alcohol entre ludópatas oscila alrededor de 15-20\%; Arbinaga (2000), señala que la prevalencia de consumo diario de alcohol es del 14,3\% entre los jugadores patológicos y Bahamón (2006) en su revisión temática, afirma que la sustancia de abuso más frecuente entre quienes tienen diagnóstico de juego patológico es el alcohol. Lo anterior permite afirmar que este hallazgo es frecuente en jugadores patológicos y obliga a ofrecer intervenciones terapéuticas que tengan presente esta situación.

Con respecto al uso de otras sustancias psicoactivas, se presenta igual situación que el alcohol, su riesgo de utilización y dependencia se encontró como factor asociado a quienes presentan juego patológico. Estudios como el de Black y Moyer (1998) encontraron que el 64\% de los jugadores patológicos tuvieron alguna vez en la vida el diagnóstico de abuso de sustancias y Kaush (2003), encontró similares prevalencias; otros autores que han tratado el tema ratifican este hallazgo, lo cual lleva a concluir la necesidad de tener en cuenta esta variable para el manejo de los pacientes con diagnóstico de jugadores patológicos (Pietrzak, Ladd y Petry, 2003; Potenza, Fiellin, Heninger, Rounsaville y Mazure, 2002; Winters, et al. 2002).

\section{AGRADECIMIENTOS}

A la Secretaria de Salud del Municipio de Medellín en Colombia y a la Universidad CES, quienes cofinanciaron la realización de este estudio.

\section{REFERENCIAS}

Adlaf, E.M. y lalomiteanu, A. (2000). Prevalence of problem gambling in adolescents: findings from the 1999 Ontario Student Drug Use Survey. Canadian Journal of Psychiatry, 45, 752-755.

American Psychiatric Association. (2000). Diagnostic and Statistical Manual of Mental Disorders, Text Revision. Washington, D.C.: Autor.

American Psychiatric Association. DSM5. Recuperado el 8 de Marzo 8 de 2010, de http://www.dsm5.org/Pages/Default.aspx

Andresen, M. (2006). Governments' conflict of interest in treating problem gamblers. Canadian Medical Association Journal, News, 175, 1191-1192.

Arbinaga, F. (2000). Estudio descriptivo sobre el juego patológico en estudiantes (8-17 años): Características sociodemográficas, consumo de drogas y depresión. Adicciones, 12, 493-505. 
Ballesteros, B.P., Novoa, M.M., Caycedo, C. y García, D. del R. (2006). Análisis funcional de casos de conductas de juego en niños $y$ jóvenes de Bogotá, Colombia. Adicciones, 18, 73-86.

Bahamón, M. (2006). Juego patológico: revisión de tema. Revista Colombiana de Psiquiatría, 35, 380-399.

Becoña, E., Lorenzo, M.C. y Fuentes, M.J. (1996). Psychological Reports, 78, 635-640

Bellaire, W. y Caspari, D. (1992). Diagnosis and therapy of male gamblers in a university psychiatric hospital. Journal of Gambling Studies, 8, 143-150.

Black, D.W. y Moyer, T. (1998). Clinical features and psychiatric morbidity of subjects with pathological gambling behavior. Psychiatric Services, 49, 1434-1439.

Black, D.W. Monahan, P.O., Temkit, M.H., y Shaw, M. (2006). A family of pathological gambling. Psychiatry Research, 141, 295-303.

Bronfenbrenner, U. (1986). Ecology of family as a context of human development. Developmental Psychology, 22, 723-742.

Ciarrocchi, J.W., y Richardson, R. (1989). Profile of compulsive gamblers in treatment: Update and comparisons. Journal of Gambling Behavior, 5, 53-65.

DANE-Departamento Nacional de Estadística. Proyecciones de poblaciones municipales por sexo y edades simples de 0-24 años. Recuperado el 9 de Marzo de 2009, de http://www.dne. gov.co

Echeburúa, E., Baez, C., Fernandez-Montalvo, J. y Paez, D. (1994). Cuestionario de juego patológico de South Oaks (SOGS): Validación española. Análisis y Modificación de Conducta, 20, 769-791.

Echeburúa, E. (2005). Retos de futuro en el tratamiento del juego patológico. Adicciones, 17, 11-16.

García, J.L., Díaz, C. y Aranda, J.A. (1993). Trastornos asociados al juego patológico. Anales de Psicología, 1, 83-92.

George, D., y Mallery, P. (1995). SPSS/PC+ step by step: A simple guide and reference. Belmont, CA: Wadsworth Publishing Company.

George, S., y Murali, V. (2005). Pathological gambling: and overview of assessment and treatment. Advances in Psychiatric Treatment, $11,450-456$.

Griffiths, M. (2004). Betting your life on it. British Medical Journal, 329, 1055-1056.

Gutiérrez, F. Juegos de azar casinos y máquinas tragamonedas. Recuperado el 23 de mayo de 2010, de http://www.federicogutierrez. com/web2010/assets/nuestroTrabajo/Descargas/CasinosTragamonedas.pdf

Huang, J-H. y Boyer, R. (2007). Epidemiology of youth gambling problems in Canada: A National Prevalence Study. Canadian Journal of Psychiatry, 52, 657-665.

Ibáñez, A., Blanco, C., Donahue, E., Lesieur, H., Pérez de Castro, I., Fernández-Piqueras, J., et al. (2001). Psychiatric comorbidity in pathological gamblers seeking treatment. American Journal Psychiatric, 158, 1733-1735.

Jacobs, D.F. (1986). A general theory of Addictions: A new theoretical model. Journal of Gambling Behavior, 2, 15-3.

Jacobs, D.F. (2000). Juvenile gambling in North America: an Analysis of long term trends and future prospects. Journal of Gambling Studies, 16, 119-152.

Kaminer, V. y Petry, N.M. (1999). Gambling behavior in youths: why we should be concern. Psychiatric Services, 50, 167-168.
Kausch, O. (2003). Patterns of substance abuse among treatmentseeking pathological gamblers. Journal of Substance Abuse Treatment, 25, 263-270.

Ko, C.H., Yen J.Y., Chen C.C., Chen S.H. y Yen C.F. (2005). Gender differences and related factors affecting online gaming addiction among Taiwanese adolescents. Journal of Nervous and Mental Disease, 193, 273-277.

Korn, D. y Shaffer, H. (1999). Gambling and the health of the public: adopting a public health perspective. Journal of Gambling Studies, 15, 289-365.

Kovacs, M. (1985). The Children's Depression Inventory (CDI). Psychopharmacology Bulletin, 21, 995-998.

Lesieur, H.R. y Blume, S.B. (1987). The South Oaks Gambling Screen (SOGS): A new instrument for the identification of pathological gamblers. American Journal of Psychiatry, 144, 1184-1188.

Lesieur, H.R. y Blume, S.B. (1993). Revising the South Oaks Gambling Screen in different settings. Journal of Gambling Studies, 9, 213-223.

Lobo, D. y Kennedy J.L. (2006). Genetic aspects of pathological gambling: a complex disorder with shared genetic vulnerabilities. Addiction, 104, 1454-1465.

Maya, J.M., Torres, Y., Murrelle, L., Rodríguez, M., Mejía, R., Gaviria, D., et al. (2000). Consumo de sustancias psicoactivas y otros factores asociados con delincuencia juvenil. Medellin, Colombia: Universidad CES.

McLeroy, K.R., Bibeau, D., Steckler, A. y Glanz, K. (1988). An ecological perspective on health promotions programs. Health Education Quarterly, 15, 351-377.

Messerlian, C., Derevensky, J. y Gupta, R. (2005). Youth gambling problems: a public health perspective. Health Promotion International, 20, 69-79.

Muñoz-Molina, Y. (2008). Meta-análisis sobre juego patológico 19972007 (2008). Revista Salud Pública, 10,150-159.

Pietrzak, R.H., Ladd, G.T. y Petry, N.M. (2003). Disordered gambling in adolescents: epidemiology, diagnosis and treatment. Pediatric Drugs, 5, 583-595.

Potenza, M.N., Fiellin D.A., Heninger G.R., Rounsaville B.J. y Mazure, C.M. (2002). Gambling: an addictive behavior with health and primary care implications. Journal of General Internal Medicine, 17, 721-732.

Reynolds, C.R. y Richmond, B.O. (1978). What I think and feel: A revised measure of children's manifest anxiety. Journal of Abnormal Child Psychology, 6, 271-280.

Shaffer, H.J., Hall, M.N. y Vander Bilt, J. (1996). Estimating the prevalence of disordered gambling behavior in the United States and Canada: a research synthesis. American Journal of Public Health, 89, 1369-1376.

Shaffer, H.J. (2003). A public Health Perspective on Gambling: the four principles. AGA Responsible Gaming Series, 2, 1-28.

Stinchfield, R.D. y Winters, K.C. (1998). Adolescent gambling: A review of prevalence, risk factors and health implications. Annals of the American Academy of Political and Social Science, 556, 172-185.

Stinchfield, R. (2002). Reliability, validity, and classification accuracy of the South Oaks Gambling Screen (SOGS). Addictive Behaviors, 27, 1-19.

Tarter, R. (1990). Evaluation and treatment of adolescent substance abuse: a decision tree method. American Journal of Drug and Alcohol Abuse, 16, 1-46. 
Tarter, R., Kirisci, L. y Mezzich, A. (1996). The Drug Use Screening Inventory: School adjustment correlates of substance abuse. Measurement and Evaluation in Counseling and Development 29, 25-34.

Torres, Y., Osorio J.J., López P.L. y Mejia R. (2006). Salud Mental del Adolescente, Medellin 2006. Medellin, Colombia: Universidad CES.

Welte, J., Wieczorek, W., Barnes, G. y Tidwell, M. (2006). Multiple risk factors for frequent and problem gambling: individual, social and ecological. Journal of Applied Psychology, 6, 1548-1568.

Westphal, J.R. y Johnson, L.J. (2003). Gender differences in psychiatric comorbidity and treatment-seeking among gamblers in treatment. eGambling, Issue 8. Recuperado el 15 de Marzo de 2010, de http://www.camh.net/egambling/archive/pdf/EJGIIssue8/EJGI8-research-westphal.pdf
Westphal, J.R., Rush, J.A., Stevens, L. y Johnson, L.J. (2000). Gambling behavior of Louisiana students in grades 6 through 12 . Psychiatric Services, 51, 96-99.

WHO-CIDI, The World Health Organization-Composite International Diagnostic Interview, (1990). Recuperado el 9 de Marzo de 2009, de http://www.hcp.med.harvard.edu/wmhcidi/ftpdir_public/ papi_instrument/papiV7/PAPIv7_Suicidality.pdf

Winters, K.C., Stinchfield, R.D., Botzet, A. y Anderson N. (2002). A prospective study of youth gambling behaviors. Psychology of Addictive Behaviors, 16, 3-9.

Vitaro, F., Arseneault, L. y Tremblay, R.E. (1997). Dispositional predictors of problem gambling in male adolescents. American Journal of Psychiatry, 154, 1769-1770. 
